\title{
A Comparative Evaluation of Shear Bond Strength of Resin-modified Glass lonomer and Zirconomer Incorporated with 1.5\% Doxycycline: An In Vitro Study
}

\author{
K. Sudha ${ }^{1}$, T. H. Kotaiah², O. Seema Reddy ${ }^{1}$, P. Sravana Laxmi ${ }^{1}$, Ch. Laxman Rao', \\ T. Leela Naga Pavani ${ }^{1}$ \\ ${ }^{1}$ Department of Conservative Dentistry and Endodontics, Government Dental College and Hospital, Vijayawada, Andhra Pradesh, \\ India, ${ }^{2}$ Department of Pediatric Dentistry, Government Dental College and Hospital, Vijayawada, Andhra Pradesh, India
}

Email for correspondence: sravanipenumaka7@gmail.com

\begin{abstract}
Context: Dental caries are the most pervasive microbiological disease in the world. To overcome this, various restorative materials were introduced. However, secondary caries have been quoted as one of the most common causes of restoration failures. Following the bacterial attack, a group of enzymes called matrix metalloproteinases (MMPs) are engaged with the degradation of the dentinal matrix. The use of tetracyclines and their derivatives with restorative could contribute to inactivate MMPs and arrest caries progression. Aim: The purpose of the present in vitro study was to evaluate the shear bond strength of resin-modified glass ionomer cement (RMGIC) and Zirconomer incorporated with 1.5\% Doxycycline. Materials and Methods: Forty freshly extracted human molars without any defects were collected. The teeth were mounted in plastic rings of 1-inch diameter with cold cure acrylic resin, and the occlusal surfaces were ground flat using a diamond disc with a water coolant at slow speed to expose the dentin. The specimens were then divided into two groups with 20 specimens in each group according to the restorative material used. The restorative materials were mixed with $1.5 \%$ Doxycycline, condensed into cylindrically shaped plastic tubes with an internal diameter of $3 \mathrm{~mm}$ and a height of $4 \mathrm{~mm}$. In Group A, the samples were light cured for $40 \mathrm{~s}$. Shear bond strength of all the samples was tested under the universal testing machine. Statistical Analysis: The results were compared using unpaired " $t$ "-test to detect significance between groups using SPSS 17.0 software. Results: Statistical analysis showed that RMGIC with $1.5 \%$ Doxycycline shows highest shear bond strength $(P<0.05)$ when compared to Zirconomer with 1.5\% Doxycycline. Conclusion: RMGIC with 1.5\% Doxycycline showed highest shear bond strength when compared to Zirconomer with $1.5 \%$ Doxycycline.
\end{abstract}

Key words: Doxycycline, glass ionomer cement, resin-modified glass ionomer, Zirconomer

\section{INTRODUCTION}

Dental materials have dynamically evolved from metallic materials to ones that could exactly

\begin{tabular}{|c|c|}
\hline Quick Response Code & Article Info: \\
\hline & doi: $10.5866 / 2017.9 .10159$ \\
\hline & $\begin{array}{l}\text { Received: } 11-08-2017 \\
\text { Revised: } 08-09-2017 \\
\text { Accepted: } 14-09-2017 \\
\text { Available Online: } 27-11-2017 \text { (www.nacd. } \\
\text { in)@ NAD, } 2017 \text { - All rights reserved }\end{array}$ \\
\hline
\end{tabular}

mimic natural tooth in appearance. Restorative dentistry is a blend of science and art. The triumph of restorative dentistry is based on the functional and esthetic results of a given mediation. Regardless of the development of various materials, the glass ionomer cement (GIC), due to its anticaries effect and ability to release fluoride still remains the routinely used material in dentistry. GIC was one of the first esthetic restorative materials introduced in the dental arena by Wilson and Kent in 1972..$^{[1,2]}$ The conventional GICs have many attractive features such as adhesion to tooth structure, slow release 
of fluoride which may produce a cariostatic action, good biocompatibility, and a shade similar to that of the tooth. The disadvantages are susceptibility to moisture contamination or dehydration during the early stages of setting, slow rate of setting. Furthermore, GICs have low fracture toughness and poor resistance to wear. ${ }^{[3,4]}$ To conquer this, several modifications in conventional GICs have been done.

Resin-modified GICs (RMGICs) have been developed to improve the mechanical properties. These materials exhibit higher moisture resistance, higher fracture toughness, better wear resistance, and a longer working time. Conventional GICs set through slow acid-base reaction whereas RMGIC initially set through resin polymerization from exposure to visible light, followed by additional hardening through an acid-base reaction. ${ }^{[5,6]}$

Zirconia (ZrO2) infused GIC (Zirconomer) has been introduced to the GIC family to address all the issues that have plagued the conventional glass ionomer thus far. As indicated by the manufacturer, Zirconomer has the quality and solidness of amalgam with the defensive advantages of glass ionomer while totally disposing of the peril of mercury. ${ }^{[7,8]}$

Studies have demonstrated that dental caries are caused by microorganisms. After caries excavation, some amount of residual bacteria still remains within the tooth. Hence, various antimicrobials have been added to glass ionomers to combat the residual bacteria. After the bacterial invasion, a group of enzymes called matrix metalloproteinases (MMPs) are involved in the dentinal matrix degradation. The incorporation of antimicrobials with an inhibitory effect on these enzymes could additionally repress dentinal breakdown along with its antibacterial effect. ${ }^{[9]}$

Tetracyclines come under the family of antibiotics which inhibit the catalytic activity of human collagenases and gelatinases, especially MMPs. The use of tetracyclines in restorative materials could inactivate caries as well as inhibit these enzymes involved in dentin degradation. ${ }^{[10]}$

Therefore, the present study was undertaken to evaluate the shear bond strength of RMGIC and Zirconomer incorporated with 1.5\% Doxycycline.

\section{MATERIALS AND METHODS}

Forty extracted human molars without any defects were collected, mounted in plastic rings of 1-inch diameter with cold cure acrylic resin, and the occlusal surfaces were ground flat using a diamond disc with a water coolant at slow speed to expose the dentin. The specimens were then divided into two groups with 20 specimens in each group according to the restorative material used.

In Group 1, the exposed dentin surfaces were conditioned with GC cavity conditioner for a duration of $10 \mathrm{~s}$, washed and air dried but not desiccated. RMGIC powder mixed with $1.5 \%$ Doxycycline, samples were incorporated with mixed material using cylindrically shaped plastic tubes with an internal diameter of $3 \mathrm{~mm}$ and a height of $4 \mathrm{~mm}$. The material was then light-cured for $40 \mathrm{~s}$ vertically and also cured for $10 \mathrm{~s}$ horizontally at 90 angles to ensure complete polymerization of the material. For each sample, the curing tip was placed as nearly as conceivable to the glass ionomer and dentin surface.

In Group 2, the exposed dentin surfaces were conditioned with GC cavity conditioner for a duration of 10 seconds washed, air dried but not desiccated. Zirconomer powder blended with $1.5 \%$ Doxycycline, condensed into circular shaped plastic tubes with an internal diameter of $3 \mathrm{~mm}$ and a height of $4 \mathrm{~mm}$.

All specimens were stored in distilled water at $37 \mathrm{C}$ for $24 \mathrm{~h}$. The shear bond strength was then determined using universal testing machine at a crosshead speed of $1 \mathrm{~mm} / \mathrm{min}$ utilizing a device constructed to direct the shearing force on the dentin to restorative material interface.

\section{Statistical analysis}

The results were compared using unpaired " $t$ "-test to detect significance between groups using SPSS 17.0 software.

\section{RESULTS}

Results showed that RMGIC with 1.5\% Doxycycline shows highest shear bond strength when compared to Zirconomer with $1.5 \%$ Doxycycline which is clinically significant $(P<0.05)$ (Table 1 and Graph 1$)$.

\section{DISCUSSION}

Dental caries are the most pervasive noncommunicable disease in the world. Tooth loss due to extraction is mainly due to extensive caries with loss of the maximum amount of tooth structure and its sequelae including failed conservative procedures. ${ }^{[11]}$ The triumph of restorative dentistry 


$\begin{aligned} & \text { Table 1: Mean shear bond strength values between the } \\
& \text { groups }\end{aligned}$
\begin{tabular}{lccc} 
Group & N & Mean \pm SD & P value \\
\hline Group 1 & 20 & $9.77223 \pm 0.48861$ & $<0.005^{*}$ \\
Group 2 & 20 & $6.50762 \pm 0.32538$ & \\
\hline
\end{tabular}

$* P<0.05$ statistically significant, $P>0.05$ non significant, NS

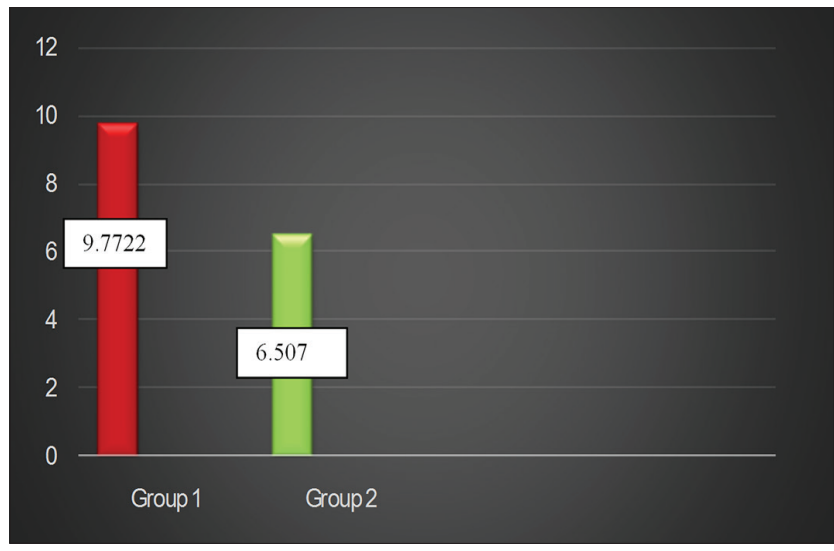

Graph 1: Graphical representation of the comparison of mean shear bond strength values between the groups

is based on the functional and esthetic results of a given intervention. ${ }^{[1]}$ Amalgam is the most commonly used restorative material in dentistry from so many years. However, hazards of mercury from amalgam restorations on human health and environment have impelled the development of new restorative materials. ${ }^{[12]}$

GIC was one of the first esthetic restorative materials introduced in the dental arena by Wilson and Kent in 1972..$^{[1,2]}$ The conventional GICs have many attractive features such as adhesion to tooth structure, slow release of fluoride which may produce a cariostatic action, good biocompatibility, and a shade similar to that of the tooth. The disadvantages are the susceptibility to moisture contamination or dehydration, slow rate of setting during the early stages of setting. Furthermore, conventional GICs have low fracture toughness and poor resistance to wear. To overcome this, several modifications in conventional GICs have been done.

RMGICs have been developed to improve the mechanical properties. RMGICs exhibit better wear resistance, higher moisture resistance, higher fracture toughness, and a longer working time. Conventional GICs set through slow acid-base reaction whereas RMGICs initially set through resin polymerization from exposure to visible light, followed by additional hardening through an acidbase reaction. ${ }^{[5,6]}$ In this study, Fuji II LC was used. It is a light curable GIC which has smaller glass particles which allow greater density and assures a smoother and glossier finish. The resin components in the liquid assume a key role in the improvement of the physical properties of the RMGICs. After initial setting, these cement allow the passage of the pulpal fluid through an absorption layer, formed near the dentinal tubules. This layer can make up for the polymerization shrinkage of the resin agent, maintaining the marginal seal of the restoration. Moreover, the polymerization stresses developed by RMGICs are much lower than the ones produced by composite resin, due to their slow setting reaction, and smaller resin content in the material. ${ }^{[13]}$

The marginal integrity or adaptation of RMGIC to the cavity walls is slightly better than Fuji II. Fuji II LC has shown lesser microleakage than Fuji IX and Fuji II. The finding of less microleakage with RMGICs is due to the fact that they have substantially better adaptation to dentin than conventional GICs. One possible explanation is that the ability of RMGICs to adhere immediately to dentin compared with the chemically cured conventional GICs that develop adhesion over time..$^{[14,15]}$

With the decrease in notoriety of amalgam in recent years, there is a need for an equally strong yet safer substitution. Zirconomer is a zirconiareinforced GIC. It is also called as "white amalgam" as it has the beneficial properties of both amalgams (compressive strength) as well as GIC (bonding and fluoride release). The manufacturer claims that the inclusion of zirconia fillers in the glass component of Zirconomer reinforces the structural integrity of the restoration and imparts higher compressive strength, good dimensional stability, and lower creep. ${ }^{[8]}$ In this study, Zirconomer was used as it is a relatively new material available, there are no studies related to bond strength with this material comparing it with other GICs.

Secondary caries have been quoted as one of the most common causes of restoration failures. Available literature reveals that approximately $60 \%$ of total replacements of restorations are due to secondary caries. In the process of supplanting existing restoration, the size of restoration changes considerably. The ability of dental restorative materials to inhibit recurrent caries is the important clinical property. ${ }^{[7]}$ However, after caries removal, many microorganisms can remain in the dentin 
substrate, even in the presence of a standard sealing. Following the bacterial attack, a group of enzymes called MMPs are engaged with the degradation of the dentinal matrix. Diverse methodologies have been described in the literature such as adding antibacterial agents to dental materials, to provide residual infection control. Indeed, antibacterial treatment of the dentin can smother the growth of remaining bacteria under existing restorations, in this way limiting the risk of recurrent caries and damage to the pulp.

Tetracyclines are a class of antibiotics which inhibit the catalytic activity of human collagenases and gelatinases, especially MMPs. The choice of Doxycycline hyclate in the current study is based on this property because caries progression is not only dependent on the bacterial activity yet in addition related to the release of MMPs from dentin that may cause speeding up of dentin destruction. They are specific inhibitors of prokaryotic ribosome, blocking receptors in $30 \mathrm{~s}$ subunit that bind to t-RNA, hindering the synthesis of protein. Human cells are not affected by tetracyclines because eukaryotic ribosomes are basically not the same as prokaryotic ones structurally. Along these lines, they are considered safe to human cells and effective against Gram-positive and Gram-negative, anaerobic, and aerobic bacteria. Another property of tetracyclines is to inhibit both activity and secretion of MMPs. Studies have shown their activity on MMP-1, MMP-2, and MMP-12 both in vitro and in vivo. Mechanism of caries is thought to be interceded by both bacterial and human proteases. In the caries process, following the disintegration of hydroxyapatite, the collagenous network becomes exposed to enzymatic degradation. Host-derived proteolytic enzymes, such as MMPs in dentin and saliva, have important role in dentin organic matrix degradation. Hence, the use of tetracyclines and their derivatives could contribute to inactivate MMPs and arrest caries progression. ${ }^{[9]}$

GICs containing $1.5 \%$ antibiotics produced bond strengths similar to those of the control GICs and do not change the properties of GICs, but a significant reduction in bond strength was observed for GICs containing $3 \%$ and $4.5 \%$ antibiotics. Presumably, the lower bond strength results from interference in the polar and ionic attraction between the carboxylate and inorganic ions with dentin and another possible explanation for low bond strength is that the increasing concentration of antibiotic powders may decrease the reaction between the glass particles and liquid cement, thereby increasing the number of unreacted particles in the structure. ${ }^{[16]}$ Hence, in this study, $1.5 \%$ Doxycycline was used.

In this study, Fuji II LC with $1.5 \%$ Doxycycline showed highest mean shear bond strength as compared to Zirconomer with $1.5 \%$ Doxycycline $(P<0.05)$. This is in accordance with several researchers confirming the higher bond strength values of RMGICs as compared to other GICs. There was postulation of hybrid layer formation of approximately 6 microns thickness between Fuji II LC and conditioned dentin. ${ }^{[17]}$ The mechanical properties of the GICs are significantly dependent on the cross-linking formation during setting. The setting reaction of GICs is an acid-base reaction forming a salt hydrogel which acts as the binding matrix within which the glass or zirconia is a filler. Upon mixing the acid with the powder, the acid attacks the powder, releasing metal ions. Adhesive failures are observed with Zirconomer which could be probably due to the interference of zirconia fillers in bonding to tooth structure. ${ }^{[18]}$ However, further studies are needed regarding various properties of Zirconomer.

\section{CONCLUSION}

Within limitations of the present study, it can be concluded that RMGIC with $1.5 \%$ Doxycycline showed highest shear bond strength when compared to Zirconomer with $1.5 \%$ Doxycycline.

\section{REFERENCES}

1. Prabhakar AR, Kalimireddy PL, Yavagal C, Sugandhan S. Assessment of the clinical performance of zirconia infused glass ionomer cement. An in vivo study. Int J Oral Health Sci 2015;5:74-9

2. Wilson AD, Kent BE. A new translucent cement for dentistry. The glass ionomer cement. Br Dent J 1972;132:133-5.

3. Miyazaki M, Iwasaki K, Onose H, Moore BK. Resinmodified glass-ionomers: Effect of dentin primer application on the development of bond strength. Eur J Oral Sci 1999;107:393-9.

4. Morabito A, Defabianis P. The marginal seal of various restorative materials in primary molars. J Clin Pediatr Dent 1997;22:51-4.

5. Ellacuria J, Triana R, Soler I, Minguez N, Prado J, Cearra P, et al. Development of glass ionomers. Ital J Paediatr Dent 1999;2:59-66.

6. Mejare SH. Conventional versus resin-modified glassionomer cement for class II restorations in primary molars. A 3-year clinical study. Int J Paediatr Dent 2003;13:2-8.

7. Tiwari S, Kenchappa M, Bhayya D, Gupta S, Saxena S, Satyarth S, et al. Antibacterial activity and fluoride 
release of glass-ionomer cement, compomer and zirconia reinforced glass-ionomer cement. J Clin Diagn Res 2016;10:90-3.

8. Zirconomer Zirconia Reinforced Restorative. Available from: $\quad$ http://www.shofu.com.sg/downloads/pdf/ ZirconomerBrochure.pdf. [Last cited on 2016 Jan 04].

9. Castilho AR, Duque C, Negrini TC, Sacono NT, De Paula AB, Sacramento PA, et al. Mechanical and biological characterization of resin-modified glass-ionomer cement containing doxycycline hyclate. Arch Oral Biol 2012;57:131-8

10. Golub LM, Ramamurthy NS, McNamara TF, Greenwald RA Rifkin BR. Tetracycline's inhibit connective tissue breakdown: New therapeutic implications for an old family of drugs. Crit Rev Oral Biol Med 1991;2:297-322.

11. Swathi CL. Evaluation of compressive strength for a combination of glassionomer cement and antibiotics. J Orofac Res 2013;3:245-8.

12. Faraj BM, Mohammad HM, Mohammad KM. The changes in dentists' perception and patient's acceptance on amalgam restoration in kurdistan-iraqa questionnaire based crosssectional study. J Clin Diagn Res 2015;9:22-5.
13. Mitsuhashi A, Hanaoka K, Teranaka T. Fracture toughness of resin-modified glass ionomer restorative materials: Effect of powder/liquid ratio and powder particle size reduction on fracture toughness. Dent Mater 2003;19:747-57.

14. Diwanji A, Dhar V, Arora R, Madhusudan A, Rathore AS Comparative evaluation of micro leakage of three restorative glass ionomer cements: An in vitro study. J Nat Sci Biol Med 2014;5:373-7.

15. Xie D, Brantley WA, Culbertson BM, Wang G. Mechanical properties and microstructures of glass-ionomer cements. Dent Mater 2000;16:129-38.

16. YesilyurtC,ErK, Tasdemir T,Buruk K, Celik D.Antibacterial activity and physical Properties of glass-ionomer cements containing antibiotics. Oper Dent 2009;34:18-23.

17. Burrow MF, Nopnakeepong U, Phrukkanon S. A comparison of microtensile bond strengths of several dentin bonding systems to primary and permanent dentin. Dent Mater 2002;18:239-45.

18. Gu YW, Yapc AU, Cheanga P, Khord KA. Zirconia-glass ionomer cement - A potential substitute for miracle mix. Scr Mater 2005;52:113-6 